Pacific Journal of Mathematics

DENDRITIC COMPACTIFICATIONS OF CERTAIN DENDRITIC 


\section{DENDRITIC COMPACTIFICATIONS OF CERTAIN DENDRITIC SPACES}

\section{B. J. Pearson}

A dendritic space is a connected space in which every two points are separated by a third point. In this paper we describe a very natural method for obtaining a dendritic compactification of any connected space for which a dendritic compactification exists. The method is an extension of the familiar process of compactifying $E^{1}$ by adjoining $-\infty$ and $+\infty$.

In what follows, an arc is a Hausdorff continuum with only two noncut points. A ray is an arc minus one of its noncut points. The space $X$ is semi-locally connected at the point $p$ if each open set containing $p$ contains an open set $V$ containing $p$ such that $X-V$ has at most finitely many components.

Lemma. If the space $X$ is arcwise connected but is not semilocally connected at the point $p$, then there exists an open set $U$ containing $p$ such that if $V$ is an open set containing $p$ and lying in $U$, then $X-V$ has infinitely many components that intersect both $\bar{V}$ and $X-U$.

Proof. There exists an open set $U$ containing $p$ such that for each open set $V$ containing $p$ and lying in $U, X-V$ has infinitely many components. Let $V$ be an open set containing $p$ and lying in $U$, and let $\mathscr{S}$ be the collection of all components of $X-V$ that intersect both $\bar{V}$ and $X-U$. Suppose $\mathscr{S}$ is finite. Let $W$ be the union of $V$ and all components of $X-V$ lying in $U$. It follows from the arcwise connectivity of $X$ that each component of $X-V$ intersects $\bar{V}$. Therefore $W=X-\cup \mathscr{S}$, so that $W$ is open. But

$$
W \cong U, X-W=\cup \mathscr{S},
$$

and $C$ is a component of $X-W$ if and only if $C \in \mathscr{S}$. Therefore $\mathscr{S}$ is infinite.

THEOREM 1. If the connected space $X$ has a dendritic compactification, then $X$ is arcwise connected and semi-locally connected.

Proof. Suppose $X$ has a dendritic compactification $X^{*}$. Since $X^{*}$ is a dendritic continuum, it is locally connected, and it then follows from Theorem 7.1 of [3] that the interval $a b$ of $X^{*}$, which consists 
of $a$ and $b$ and the set of all points of $X^{*}$ separating $a$ from $b$, is an arc. Suppose $a b$ contains a point $x$ not in $X$. Then $X^{*}-\{x\}$ is the union of two disjoint open sets $U$ and $V$ such that $a \in U$ and $b \in V$. But then $X$ is a connected subset of $U \cup V$, so that $X \subseteq U$ or $X \subseteq V$. Therefore $a b \subseteq X$.

Suppose $X$ is not semi-locally connected at $p$. There exist open sets $U$ and $V$ in $X^{*}$ such that $p \in V \subseteq \bar{V} \subseteq U$ and an infinite net $\left\{C_{\alpha}\right\}$ of distinct components of $X-V$ intersecting both $\bar{V}$ and $X-U$, where the closures are taken in $X^{*}$. For each $\alpha$ let $x_{\alpha} \in C_{\alpha} \cap \bar{V}$. Some subnet $\left\{x_{\alpha_{n}}\right\}$ of $\left\{x_{\alpha}\right\}$ converges to a point $x$ in $X^{*}$. For each $n$ let $y_{\alpha_{n}} \in C_{\alpha_{n}} \cap(X-U)$. Since $X^{*}$ is compact, the net $\left\{y_{\alpha_{n}}\right\}$ has a cluster point $y$ in $X^{*}$. Now $x \in \bar{V}$ and $y \in X^{*}-U$, so that $x \neq y$. But then no point separates $x$ from $y$ in $X^{*}$.

THEOREM 2. If the space $X$ is dendritic, semi-locally connected, and arcwise connected, and each ray in $X$ is a subset of some arc in $X$, then $X$ is compact.

Proof. Let $p \in X$. Suppose $\left\{x_{\alpha}\right\}$ is a net of points in $X-\{p\}$ with no cluster point. Suppose that for each $\alpha$ and each point $x$ of the arc $p x_{\alpha}$ different from $p$ there is a $\beta>\alpha$ such that $x \notin p x_{\beta}$. Let $U$ be an open set containing $p$ such that for each $\alpha$ there is a $\beta>\alpha$ such that $x_{\beta} \notin U$. There is an open set $V$ containing $p$ and lying in $U$ such that $X-V$ has at most finitely many components. There is an $\alpha_{0}$ such that $x_{\alpha_{0}} \in X-V$. Let $x_{0} \in p x_{\alpha_{0}}$ such that $p x_{0} \subseteq V$. There is an $\alpha_{1}>\alpha_{0}$ such that $x_{\alpha_{1}} \in X-V$ and $x_{0} \notin p x_{\alpha_{1}}$. Let $x_{1} \in p x_{\alpha_{1}}$ such that $p x_{1} \subseteq V$. There is an $\alpha_{2}>\alpha_{1}$ such that $x_{\alpha_{2}} \in X-V, x_{0} \notin p x_{\alpha_{2}}$, and $x_{1} \notin p x_{\alpha_{2}}$. Continue this process. There exist $m$ and $n$ such that $m \neq n$ and some component of $X-V$ contains both $x_{\alpha_{m}}$ and $x_{\alpha_{n}}$. But then no point of $X$ separates $x_{\alpha_{m}}$ from $x_{\alpha_{n}}$. This is a contradiction, and hence the set $R$ of all points $x$ in $X-\{p\}$ such that for some $\alpha, x \in p x_{\beta}$ for each $\beta>\alpha$ is nonempty. Let $x, y \in R$. There is an $\alpha_{1}$ such that $x \in p x_{\beta}$ for $\beta>\alpha_{1}$. There is an $\alpha_{2}$ such that $y \in p x_{\beta}$ for $\beta>\alpha_{2}$. Hence if $\beta>\alpha_{1}, \alpha_{2}$, then $x, y \in p x_{\beta}$. It follows that if $x, y \in R$, then either $p x \subseteq p y$ or $p y \subseteq p x$. Therefore there exists a point $q$ of $X$ distinct from $p$ such that $R=p q$ or $R=p q-\{q\}$. For each $\alpha$ let $y_{\alpha}$ be the last point of $p x_{\alpha}$ on $p q$. Let $U$ be an open set containing $q$. There is a point $y$ such that $p y q$ and $y q \subseteq U$. Since $y \in R$, there is an $\alpha$ such that if $\beta>\alpha$, then $y \in p x_{\beta}$. Hence $y_{\beta} \in y q$ for $\beta>\alpha$, so that the net $\left\{y_{\alpha}\right\}$ converges to $q$. It follows that there is a subnet $\left\{y_{\alpha_{n}}\right\}$ of $\left\{y_{\alpha}\right\}$ converging to $q$ such that if $m<n$, then $y_{\alpha_{m}}$ precedes $y_{\alpha_{n}}$ on $p q$ and $x_{\alpha_{n}} \notin p q$. There exists an open set $V$ containing $q$ and 
lying in $U$ such that $X-V$ has only finitely many components. Hence there is an $m$ such that if $n>m$, then $x_{\alpha_{n}} \in V$. It follows that $q$ is a cluster point of $\left\{x_{\alpha}\right\}$.

An incorrect version of the following lemma is stated as Lemma 3 to Theorem 3 in [1]. The lemma stated here may be used as a substitute without altering the proof of that theorem.

LEMma. If $H$ and $K$ are two separated connected sets in the arcwise connected dendritic space $X$, then some point of $X$ separates $H$ from $K$.

Proof. Let $a \in H$ and $b \in K$. Since $H$ and $K$ are separated, there exists a point $p$ of the arc $a b$ not in $H \cup K$. Let $U$ be the set of all points $x \neq p$ such that $p x$ contains a point of $a p-\{p\}$, and let $V=X-(U \cup\{p\})$. Suppose the point $x$ of $U$ is a limit point of $V$. Then there exists a net $\left\{x_{\alpha}\right\}$ of points in $V-p b$ converging to $x$ and a net $\left\{y_{\alpha}\right\}$ of points in $p b$ such that for each $\alpha, y_{\alpha}$ is the last point of $p b$ on $p x_{\alpha}$. Some point $y$ of $p b$ is a cluster point of $\left\{y_{\alpha}\right\}$, and hence no point of $X$ separates $x$ from $y$. This is a contradiction. Therefore $U$ is open, and it follows by a similar argument that $V$ is open. Since $H$ and $K$ are connected, $H \subseteq U$ and $K \subseteq V$. Therefore $p$ separates $H$ from $K$.

THEOREM 3. The dendritic space $X$ has a dendritic compactification if and only if $X$ is arcwise connected and semi-locally connected.

Proof. Suppose $X$ is arcwise connected and semi-locally connected. Let $p \in X$, and let $X^{*}$ be the union of $X$ and the collection of all maximal rays in $X$ starting from $p$. Let $\mathscr{S}$ be the collection of all open sets $U$ in $X$ such that $X-U$ has at most finitely many components. For each $U$ in $\mathscr{S}$ let $U^{*}$ be the union of $U$ and the collection of all maximal rays starting from $p$ and having a subray lying in $U$. Let $\mathscr{S}^{*}=\left\{U^{*} \mid U \in \mathscr{S}\right)$. It is easily seen that if $U, V \in \mathscr{S}$, then $U \cap V \in \mathscr{S}$ and $(U \cap V)^{*}=U^{*} \cap V^{*}$. Therefore $\mathscr{S}^{*}$ is a base for a topology of $X^{*}$, and $X$ with its original topology is a subspace of $X^{*}$. Now for each maximal ray $R$ in $X$ starting from $p$ the point $R$ of $X^{*}$ is a limit point of the point set $R$. Therefore $X$ is dense in $X^{*}$. Furthermore $R \cup\{R\}$ is an arc from $p$ to $R$. Therefore $X^{*}$ is arcwise connected. Suppose $U \in \mathscr{S}$ and $C$ is a component of $X^{*}-U^{*}$ containing a point $R$ of $X^{*}-X$. If $R$ has a subray in $U$, then $R \in U^{*}$. Hence there is a point $x$ in $R-U$. Let $K$ be the component of $X-U$ containing $x$. Since

$$
X-U \subseteq X^{*}-U^{*},
$$


it follows that $K \subseteq C$. Hence each component of $X^{*}-U^{*}$ contains a component of $X-U$. Therefore $X^{*}$ is semi-locally connected. Let $a$ and $b$ be points of $X$. There is a point $x$ of $X$ such that $X-\{x\}$ is the union of two disjoint open sets $U$ and $V$ in $X$ such that $a \in U$ and $b \in V$. Since the only component of $X-U$ is $V \cup\{x\}$, it follows that $U \in \mathscr{S}$ and similarly that $V \in \mathscr{S}$. Since $U \cap V=\varnothing$, it follows that $U^{*} \cap V^{*}=\varnothing$. If $R \in X^{*}-X$, then there is a subray $S$ of $R$ such that $x \notin S$. Hence $S \subseteq U$ or $S \subseteq V$, so that $R \in U^{*}$ or $R \in V^{*}$. Therefore $X^{*}-\{x\}=U^{*} \cup V^{*}$. It follows that $x$ separates $a$ from $b$ in $X^{*}$. Now let $a \in X$ and $R \in X^{*}-X$. There is a subray $S$ of $R$ such that $a \notin S$. Some point $x$ of $X$ separates $a$ from $S$ in $X$, and it follows as before that $x$ separates $a$ from $R$ in $X^{*}$. Finally, let $P$ and $R$ be two elements of $X^{*}-X$. There exist disjoint rays $Q$ and $S$ such that $Q \subseteq P$ and $S \subseteq R$. Some point $x$ of $X$ separates $Q$ from $S$ in $X$. It follows that $x$ separates $P$ from $R$ in $X^{*}$. Therefore $X^{*}$ is dendritic. It remains to be proved that $X^{*}$ is compact. Suppose $R$ is a ray in $X^{*}$ starting from a point $q$ in $X^{*}$. Now $X^{*}-X$ is totally disconnected since each two points of $X^{*}-X$ are separated by a point of $X$, and if $x$ and $y$ are points of $X$, then the arc $x y$ in $X^{*}$ is a subset of $X$. It follows that $R-\{q\} \subseteq X$. Hence there is a maximal ray $S$ in $X$ starting from $p$ and containing a subray of $R$. Since $S \cup\{S\}$ is an arc in $X^{*}, R$ is contained in some arc in $X^{*}$. It now follows from Theorem 2 that $X^{*}$ is compact. This completes the proof.

Two other methods for obtaining dendritic compactifications of dendritic spaces may be found in the literature. In [2] Ward proves, by embedding in a Tychonoff cube, that every locally connected dendritic space satisfying a certain convexity condition has a dendritic compactification. In [1] Proizvolov proves, by considering maximal collections of closed connected sets having the finite intersection property, that every locally peripherally compact dendritic space has a dendritic compactification.

\section{REFERENCES}

1. V. V. Proizvolov, Peripherally bicompact tree-like spaces, Dokl. Akad. Nauk SSSR, 189 (1969), 724-727.

2. L. E. Ward, Jr., On dendritic sets, Duke Math. J., 25 (1958), 505-514.

3. G. T. Whyburn, Cut points in gereral topological spaces, Proc. Nat. Acad. Sci. USA, 61 (1968), 380-387.

Received April 28, 1972 and in revised form July 13, 1972. 


\section{PACIFIC JOURNAL OF MATHEMATICS}

\section{EDITORS}

D. Gilbarg and J. Milgram

Stanford University

Stanford, California 94305

R. A. Beaumont

University of Washington

Seattle, Washington 98105
J. DUGUNDJI* Department of Mathematics

University of Southern California Los Angeles, California 90007

RICHARD ARENS

University of California

Los Angeles, California 90024

\section{ASSOCIATE EDITORS}

E. F. BeCKenbach

B. H. NeUMaNN

F. WOLF

K. Yoshida

\section{SUPPORTING INSTITUTIONS}

UNIVERSITY OF BRITISH COLUMBIA

UNIVERSITY OF SOUTHERN CALIFORNIA

CALIFORNIA INSTITUTE OF TECHNOLOGY

UNIVERSITY OF CALIFORNIA

MONTANA STATE UNIVERSITY

STANFORD UNIVERSITY

UNIVERSITY OF TOKYO

UNIVERSITY OF NEVADA

UNIVERSITY OF UTAH

NEW MEXICO STATE UNIVERSITY

WASHINGTON STATE UNIVERSITY

OREGON STATE UNIVERSITY

UNIVERSITY OF OREGON

OSAKA UNIVERSITY

UNIVERSITY OF WASHINGTON

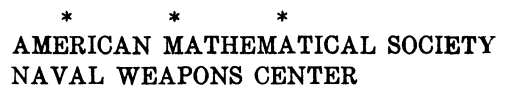

* C. DePrima will replace J. Dugundji until August 1974. 


\section{Pacific Journal of Mathematics}

\section{Vol. 47, No. $1 \quad$ January, 1973}

K. Adachi, Masuo Suzuki and M. Yoshida, Continuation of holomorphic

mappings, with values in a complex Lie group ....................

Michael Aschbacher, A characterization of the unitary and symplectic groups

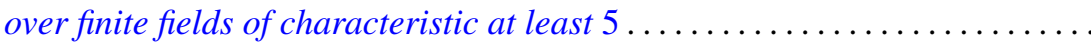

Larry Eugene Bobisud and James Calvert, Energy bounds and virial theorems for abstract wave equations....................................

Christer Borell, A note on an inequality for rearrangements ................

Peter Southcott Bullen and S. N. Mukhopadhyay, Peano derivatives and general

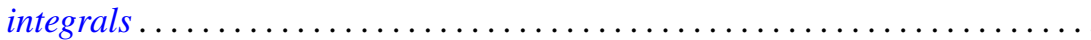

Wendell Dan Curtis, Yu-Lee Lee and Forrest Miller, A class of infinite dimensional subgroups of $\operatorname{Diff}^{r}(X)$ which are Banach Lie groups .........

Paul C. Eklof, The structure of ultraproducts of abelian groups ...............

William Alan Feldman, Axioms of countability and the algebra $C(X) \ldots \ldots \ldots$

Jack Tilden Goodykoontz, Jr., Aposyndetic properties of hyperspaces...........

George Grätzer and J. Płonka, On the number of polynomials of an idempotent algebra. II ...........................................

Alan Trinler Huckleberry, The weak envelope of holomorphy for algebras of

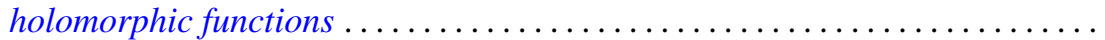

John Joseph Hutchinson and Julius Martin Zelmanowitz, Subdirect sum decompositions of endomorphism rings . . . . . . . . . . . . . . . .

Gary Douglas Jones, An asymptotic property of solutions of

$y^{\prime \prime \prime}+p y^{\prime}+q y=0$.

Howard E. Lacey, On the classification of Lindenstrauss spaces .

Charles Dwight Lahr, Approximate identities for convolution measure algebras.

George William Luna, Subdifferentials of convex functions on Banach

spaces.

Nelson Groh Markley, Locally circular minimal sets. .

Robert Wilmer Miller, Endomorphism rings of finitely generated projective modules

Donald Steven Passman, On the semisimplicity of group rings of linear

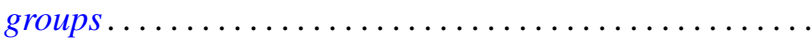

Bennie Jake Pearson, Dendritic compactifications of certain dendritic spaces.

Ryōtarō Satō, Abel-ergodic theorems for subsequences ...... .

Henry S. Sharp, Jr., Locally complete graphs. . .

Harris Samuel Shultz, A very weak topology for the Mikusinski field of

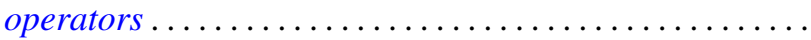

Elena Stroescu, Isometric dilations of contractions on Banach spaces ...

Charles W. Trigg, Versum sequences in the binary system ... . .

William L. Voxman, On the countable union of cellular decompositions of n-manifolds 